\title{
THEORY OF WINDS FROM COOL STARS
}

\author{
Thomas E. Holzer \\ High Altitude Observatory \\ National Center for Atmospheric Research ${ }^{*}$ \\ P. O. Box 3000 \\ Boulder, CO 80307
}

\begin{abstract}
The goal of this paper is to provide a framework for thinking about the various physical processes that may play significant roles in driving the massive winds of cool, low-gravity stars. First, some general theoretical considerations involving mass, momentum, and energy balance are discussed. Next, the value of the solar wind as an analog for these late-type stellar winds and for related astrophysical flows is briefly examined. Finally, four specific mass-loss mechanisms are discussed, and the possible importance of each of these mechanisms for massive winds from cool, low-gravity stars is evaluated.
\end{abstract}

\section{INTRODUCTION}

The two principal purposes of this review are to complement the related observational and theoretical papers presented at this conference concerning the use of the solar wind as an analog for other astrophysical flows and concerning physically meaningful ways of approaching the general mass loss problem. It is hoped that we can develop here a framework for thinking about the various physical processes that may be important in driving massive winds from cool, low-gravity stars: a framework that is useful both to researchers developing theoretical models and to those interpreting observations of these massive stellar winds. Because an extensive review of this subject was published a little more than a year ago (Holzer and MacGregor 1985), the present paper has simply been adapted from that review, with some restructuring to make it more appropriate to this conference and with some extension of the material covered to address specific questions raised at this conference. There is no attempt made here to provide a complete reference list, as that is readily available to the reader upon reference to the aforementioned review (Holzer and MacGregor 1985) and to other recent reviews (e.g., Cassinelli and MacGregor 1986; Goldberg 1984). The interested reader can find more detailed discussion of each of the four mass-loss mechanisms considered here in the review of Holzer and MacGregor (1985) and in papers referenced therein.

${ }^{*}$ The National Center for Atmospheric Research is sponsored by the National Science Foundation. 


\section{GENERAL THEORETICAL CONSIDERATIONS}

Let us consider the steady, radial, spherically symmetric expansion of a stellar atmosphere. Although non-steady flow phenomena and substantial departures from spherical symmetry can be expected to occur in all stellar winds, a study of steady, spherically symmetric expansion provides us with a basis for understanding the more complex flow systems in real stellar atmospheres. The equations describing mass, momentum, and energy balance in our simple flow system can be written

$$
\begin{gathered}
-\dot{M}_{*}=4 \pi \rho u r^{2}=\text { constant } \\
u \frac{d u}{d r}=-\frac{1}{\rho} \frac{d p}{d r}-\frac{G M_{*}}{r^{2}}+D, \\
\frac{1}{\gamma-1} p u \frac{d}{d r} \ln \left(\frac{p}{\rho^{\gamma}}\right)=-\frac{1}{r^{2}} \frac{d}{d r}\left(q r^{2}\right)+Q
\end{gathered}
$$

where $\rho, u, p$, and $q$ are the mass density, flow speed, thermal pressure, and heat flux density, $\gamma$ is the ratio of specific heats, $\rho D$ and $Q$ are the net volume rates at which momentum and heat are added to the flow, and $G, M_{*}$, and $-\dot{M}_{*}$ are the gravitation constant, stellar mass, and stellar mass loss rate. Depending on the physical processes giving rise to momentum addition $(D \neq 0)$ and heat addition $(Q \neq 0)$, we may also need Maxwell's equations, a radiative transfer equation, and other auxiliary equations to close our system of equations.

It will prove useful at times to write the energy balance description in the form [derived from eqns. (2) and (3)]

$$
F=\left(-\dot{M}_{*}\right)\left[\frac{1}{2} u^{2}+\frac{\gamma}{\gamma-1} \frac{p}{\rho}-\frac{G M_{*}}{r}\right]+4 \pi q r^{2}=F_{o}+F_{A}
$$

where the "o" subscript refers to a reference level, $r_{o}$, and

$$
F_{A}=\int_{r_{0}}^{r} d r^{\prime}\left[\left(-\dot{M}_{*}\right) D+4 \pi r^{2} Q\right]
$$

Evidently, $F$ is the energy flux of the wind associated with advection of flow energy, enthalpy, and gravitational potential energy and with the conduction of heat. Note that the energy added to the wind as it expands [cf. $F_{A}$ in eqns. (4) and (5)] results from both momentum addition and heat addition. It is most important to distinguish momentum addition from heat addition and to avoid considering energy addition and heat addition to be synonymous. These distinctions can be understood through consideration of equations (2)-(5). Such a consideration reveals that momentum addition corresponds to the addition of flow energy to the fluid through the application of a body force, while heat 
addition corresponds to the addition of internal energy to the fluid (and an increase in entropy of the fluid), generally through the dissipation of some energy flux (such as an energy flux associated with an electromagnetic radiation field or hydromagnetic waves).

\subsection{Energy requirements}

The energy-per-unit-mass of a stellar wind can be defined by [cf. eqn. (4)]

$$
E=\frac{1}{2} u^{2}+\frac{\gamma}{\gamma-1} v_{t}^{2}-\frac{1}{2} v_{g}^{2}+\frac{1}{2} v_{q}^{2}
$$

where the characteristic speeds associated with internal energy $\left(v_{t}\right)$, gravity $\left(v_{q}\right)$, and thermal conduction $\left(v_{q}\right)$ are defined by

$$
\begin{gathered}
v_{t}^{2}=p / \rho \\
v_{g}^{2}=2 G M_{*} / r \\
v_{q}^{2}=8 \pi q r^{2} /\left(-\dot{M}_{*}\right) .
\end{gathered}
$$

In the present context, it is most appropriate to interpret these characteristic speeds in terms of the energy available to the flow that resides in internal energy, gravitational potential energy, and a conductive energy flux. The gravitational contribution to the available energy is, of course, negative, because it reflects the work that must be done to lift the wind out of the gravitational field.

If we consider only stellar winds that are strongly gravitationally bound at the atmospheric base and whose energy flux is almost entirely in the form of directed flow energy at large distances from the star, then we can evaluate equation (6) at the atmospheric base $\left(r_{0}\right)$ and at a suitably large distance from the $\operatorname{star}\left(r_{\infty}\right)$ as follows

$$
\begin{gathered}
E_{o} \approx-\frac{1}{2} v_{g o}^{2}+\frac{1}{2} v_{q o}^{2} \\
E_{\infty} \approx \frac{1}{2} u_{\infty}^{2} .
\end{gathered}
$$

Evidently, the difference between $E_{\infty}$ and $E_{o}$ represents the energy-per-unit-mass that must be added to the stellar atmosphere above the atmospheric base in order to drive the wind. This energy requirement can be expressed in terms of the energy flux $F_{A}$ by making use of equations (5) and (9)-(11): 


$$
\begin{aligned}
F_{A \infty} & =\int_{r_{0}}^{r_{\infty}} d r\left[\left(-\dot{M}_{*}\right) D+4 \pi r^{2} Q\right] \\
& \approx \frac{1}{2}\left(-\dot{M}_{*}\right)\left(u_{\infty}^{2}+v_{g o}^{2}\right)-4 \pi q_{o} r_{o}{ }^{2} .
\end{aligned}
$$

If we include the conductive flux as a component of the energy flux said to be driving a stellar wind, then we can conclude that the driving energy flux, $F_{d o}$, that must be supplied at the atmospheric base $\left(r_{o}\right)$ and dissipated in the atmosphere to produce a wind characterized by $-\dot{M}_{*}$ and $u_{\infty}$ is given by

$$
F_{d o} \geq F_{A \infty}+4 \pi q_{o} r_{o}^{2} \approx \frac{1}{2}\left(-\dot{M}_{*}\right)\left(u_{\infty}^{2}+v_{g o}^{2}\right)
$$

Note that $F_{d o}$ may be larger than the nominal estimate of the driving energy flux, because some part of the energy flux supplied to the wind through heating can be radiated away (i.e., $Q$ is a net heating function, and the dissipation of the driving energy flux must account for the actual heating rate, which is given by the sum of $Q$ and the radiative cooling rate).

The information contained in equation (13) can be simply stated in words: the energy flux required to drive a stellar wind is that necessary to lift the mass transported by the wind out of the stellar gravitational field, to accelerate the wind to its asymptotic flow speed, and to supply the energy radiated away by the wind. Let us, for the moment, ignore the energy radiated by the wind, as it is generally observed to be small for the winds we consider (see, however, section 3 on thermally driven winds). In massive winds from early-type stars, $u_{\infty}^{2}>>v_{g o}^{2}$, and most of the driving energy goes into accelerating the flow to its asymptotic speed. In solar-type winds (and some hybrid-star winds), $u_{\infty}^{2} \approx v_{g o}^{2}$, and comparable parts of the wind's driving energy go into lifting the expanding atmosphere out of the stellar gravitational field and into accelerating it to its asymptotic speed. In the massive winds from low-gravity, late-type stars (except for some hybrid stars), $u_{\infty}^{2}<<v_{g o}^{2}$, and almost all of the driving energy of the wind goes in to lifting the expanding atmosphere out of the stellar gravitational field.

It is possible to use equation (13) to estimate the energy flux density emanating from the atmospheric base that is required to drive a stellar wind, provided we know the values of the stellar mass and radius $\left(M_{*}\right.$ and $\left.R_{*}\right)$ and the wind mass loss rate and asymptotic flow speed $\left(-\dot{M}_{*}\right.$ and $\left.u_{\infty}\right)$. Assuming $r_{o}=R_{*}$, and expressing $-\dot{M}_{*}$ in units of solar masses per year, equation (13) can be written

$$
\begin{aligned}
& \frac{F_{d o}}{4 \pi r_{o}{ }^{2}} \gtrsim 3.3 \times 10^{3}\left(\frac{-\dot{M}_{*}}{10^{-7}}\right)\left(\frac{M_{*}}{M_{\odot}}\right) \cdot \\
& \cdot\left(\frac{400 R_{\odot}}{R_{*}}\right)^{3}\left(1+\frac{u_{\infty}^{2}}{v_{g o}^{2}}\right) \operatorname{erg~cm}^{-2} \mathrm{~s}^{-1}
\end{aligned}
$$

The solar wind, which has a very low mass loss rate $\left(-\dot{M}_{\odot} \approx 2 \times 10^{-14} M_{\odot} y^{-1}\right)$, is found to have a driving energy flux density requirement of about $10^{5} \mathrm{erg} \mathrm{cm}^{-2} \mathrm{~s}^{-1}$. (Note that the value of $5 \times 10^{5} \mathrm{erg} \mathrm{cm}^{-2} \mathrm{~s}^{-1}$ normally quoted for high-speed solar wind streams 
takes account of the non-spherically symmetric expansion of high speed streams.) For a typical K5 supergiant $\left(M_{*} \approx 16 M_{\odot}, R_{*} \approx 400 R_{\odot}\right)$, which has a much larger mass loss rate $\left(-\dot{M}_{*} \approx 10^{-7} M_{\odot} y^{-1}\right)$ than the sun, the driving energy flux density requirement is about $6 \times 10^{4} \mathrm{erg} \mathrm{cm}^{-2} \mathrm{~s}^{-1}$, which is comparable to the solar case. Similarly, a typical Mira variable $\left(M_{*} \approx M_{\odot}, R_{*} \approx 400 R_{\odot}\right)$, which has an even larger mass loss rate $\left(-\dot{M}_{*} \approx 2 \times 10^{-6} M_{\odot} y^{-1}\right)$, has a driving energy flux density requirement of only about $5 \times 10^{4} \mathrm{erg} \mathrm{cm}^{-2} \mathrm{~s}^{-1}$, again comparable to the solar case. Why is it that these very massive winds have atmospheric base driving energy flux density requirements comparable to that of the very tenuous solar wind? First, the radii of these stars with massive winds are so large that the gravitational potential energy barrier $\left(\propto R_{*}^{-1}\right)$ is much smaller than that of the sun; second, the large stellar radii correspond to a stellar surface area $\left(\propto R_{*}^{2}\right)$ from which the driving energy flux is supplied that is much larger than the solar surface area. In other words, despite the fact that $\dot{M}_{*} / \dot{M}_{\odot} \approx 10^{7}$, this is more than made up for by the fact that $\left(R_{*} / R_{\odot}\right)^{3} \approx 6 \times 10^{7}$ [cf. eqn. (14)].

\subsection{Momentum requirements}

Consideration of the wind momentum flux has been found to place important constraints on the mass loss mechanism of for massive winds from hot stars, and for this reason some workers assume that momentum flux considerations should be equally useful in the study of massive winds from cool stars. Yet, in the case of cool stars, the usefulness is severely limited. Consider, for example, a wind driven purely by radiation pressure (above some level $r=r_{o}$ in the atmosphere), for which momentum balance is described by

$$
\frac{1}{r^{2}} \frac{d}{d r}\left[\rho u^{2} r^{2}+(1-\eta) L / 4 \pi c\right]=-\frac{\rho v_{g o}^{2} r_{o}}{2 r^{2}}
$$

Here, $\eta(r)$ measures the fraction of the asymptotic stellar radiation momentum flux $(L / c)$ received by the wind during its transit from the atmospheric reference level $\left(r_{o}\right)$ to a distance $r$. Integrating equation (15) from the reference level $\left(r_{o}\right)$ to a point in the asymptotic flow regime $\left(r_{\infty}\right)$ yields

$$
-\dot{M}_{*} u_{\infty}=\frac{\eta_{\infty} L}{c}\left(1+\frac{v_{g o}^{2}}{u_{\infty}^{2}} \frac{u_{\infty}}{2<u>}\right)^{-1}
$$

where $\langle u\rangle^{-1}=\int_{r_{0}}^{r_{\infty}} d r r_{o} / u r^{2}$. When the asymptotic flow speed is much larger than the gravitational escape speed at $r_{o}$ (more specifically, when $v_{\rho o}^{2}<<2 u_{\infty}<u>$ ), then equation (16) reduces to the more familiar form

$$
-\dot{M}_{*} u_{\infty} \approx \eta_{\infty} L / c
$$

Note that $\eta_{\infty}$ can be greater than unity when there is multiple scattering of photons and less than unity when only a fraction of the stellar radiation field couples to the 
wind, but it is frequently assumed that $\eta_{\infty} \approx 1$. Equation $(17)$ is applicable to many hot star winds, in which the flow accelerates rapidly $\left(\langle u\rangle \sum u_{\infty} / 2\right)$ and the asymptotic flow speed is much larger than the atmospheric base gravitational escape speed (viz., $u_{\infty}^{2} \gg v_{g o}^{2}$, with $r_{o}=R_{*}$ ). Its only possible applicability to cool stars, however, appears to exist when one sets the reference level far enough out in the atmosphere $\left(r_{o} \gg R_{*}\right)$ that $v_{g_{o}}^{2}<<u_{\infty}^{2}$ (also, it is necessary that $u_{o}^{2}<<u_{\infty}^{2}$ ). Then it is required that some mechanism (e.g., waves) extend the atmosphere in $R_{*} \leq r \lesssim r_{o}$ and that radiation pressure abruptly take over from the atmospheric extension mechanism, near $r=r_{o}$ and accelerate the wind to its asymptotic flow speed. Even in this very special case, it is a mistake to imagine that the wind from a star for which $v_{q_{*}}^{2}<<u_{\infty}^{2}$ is driven by radiation pressure (as might be inferred from eqn. (17)), for most of the driving energy is supplied by the atmospheric extension mechanism.

The preceding discussion indicates why one should generally avoid trying to apply momentum flux arguments to winds from cool stars, even though the same arguments have proved useful in considerations of winds from hot stars. Up to this time, it appears that such arguments have led to more confusion than clarification of our understanding of cool star winds.

\subsection{Mass loss rate and asymptotic flow speed}

There are some rather simple concepts relating to the mass flux of a stellar wind that can help us interpret observations of mass loss rates and asymptotic flow speeds. Consider first an isothermal, thermally driven wind: from equations (1) and (2), with $D=0$, we obtain

$$
\left(u^{2}-v_{t}^{2}\right) \frac{1}{u} \frac{d u}{d r}=\frac{2}{r}\left(v_{t}^{2}-\frac{1}{4} v_{g}^{2}\right)
$$

Integrating equation (18) from $r_{o}$ to the point where $u=v_{t}$ [i.e., the critical point of eqn. (18), $r_{c}=r_{o} v_{g o}^{2} / 4 v_{t}^{2}$ (Parker 1958): cf. section 2.4)

$$
-\dot{M}_{*} \propto u_{o}=v_{t}\left(\frac{v_{g o}^{2}}{4 v_{t}{ }^{2}}\right)^{2} \exp \left(-\frac{v_{g o}{ }^{2}}{2 v_{t}{ }^{2}}+\frac{3}{2} \frac{u_{o}^{2}}{2 v_{t}{ }^{2}}\right)
$$

For stellar atmospheres that are strongly gravitationally bound at the atmospheric base (i.e., $v_{g o}^{2} \gg 4 v_{t}^{2}$ ), it is clear from equation (14) (cf. leading term in argument of exponential) that the mass loss rate increases exponentially with increasing atmospheric temperature. This strong temperature dependence of $-\dot{M}_{*}$ results from the atmospheric extension produced by increasing the temperature and thus the density scale height, and it is evidently directly related to an equally strong temperature dependence of the radiative flux emanating from a thermally driven wind (cf. section 3 ).

Note that in the above discussion of mass loss rate, only the properties of the atmosphere inside the critical point (where $u=v_{t}$ in the isothermal case) are considered. More generally, we can say that the mass loss rate of a stellar wind is normally primarily determined by the properties of the atmosphere inside the sonic point of the flow (the sonic point corresponds to the critical point in the isothermal case; see, however, section 2.4), because beyond this point compressive information cannot propagate upstream in 
the wind, and thus cannot reach the atmospheric base so as to modify $u_{0}$. This statement is also normally applicable to winds with driving forces other than the thermal pressure gradient force $(D \neq 0)$, for which equation (19) can be generalized to

$$
-\dot{M}_{*} \propto u_{o}=v_{t}\left(\frac{r_{\theta}}{r_{o}}\right)^{2} \exp \left[-\frac{v_{g o}{ }^{2}}{2 v_{t}{ }^{2}}\left(1-\frac{2}{v_{g o}^{2}} \int_{r_{o}}^{r_{s}} d r D-\frac{r_{o}}{r_{\theta}}\right)-\frac{1}{2}+\frac{u_{o}{ }^{2}}{2 v_{t}{ }^{2}}\right],
$$

where $r_{\theta}$ is the location of the sonic point, at which the flow speed equals the sound speed. From equation (20) we can infer that energy addition by either heating (which increases $v_{t}$ ) or by momentum addition (corresponding to an increase in $D$ ) is effective in increasing the mass loss rate $\left(-\dot{M}_{*}\right)$ only if it occurs in the region of subsonic flow. This inference is also significant for the asymptotic flow speed of the wind, as we see below.

Recall from equation (13) that the asymptotic flow speed is simply related to the (net) energy added to the wind and to the mass loss rate:

$$
u_{\infty}^{2} \approx 2\left(F_{A \infty}+4 \pi q_{o} r_{o}^{2}\right) /\left(-\dot{M}_{*}\right)-v_{g o}^{2}
$$

Obviously [cf. eqn. (12)], $F_{A \infty}$ is increased regardless of whether energy addition to the subsonic or supersonic region of flow is increased, but (as seen above) $-\dot{M}_{*}$ is increased only if energy addition to the subsonic region is increased. As it happens, energy addition to the subsonic region tends to produce such a large increase in $-\dot{M}_{*}$, relative to the increase in $F_{A \infty}$, that $u_{\infty}$ is either unaffected or slightly decreased by subsonic energy addition. In general, we conclude that the addition of energy to the region of subsonic stellar wind flow has the effect of increasing the mass loss rate $\left(-\dot{M}_{*}\right)$ but has relatively little effect on the asymptotic flow speed $\left(u_{\infty}\right)$, while the addition of energy to the region of supersonic flow increases $u_{\infty}$ but has relatively little effect on $-\dot{M}_{*}$ (e.g., Leer and Holzer 1980). For a wind in which $u_{\infty}^{2}<<v_{g o}^{2}$, most of the driving energy of the wind must, therefore, be supplied in the region of subsonic flow, and it is to this region where we must direct most of our attention when discussing the driving mechanisms of massive winds from cool stars.

\subsection{Critical points}

Often (e.g., in discussions on the fourth day of this conference), much is made of the critical point(s) of the stellar wind equation [e.g., eqn . (18)] in discussions of the physics of stellar winds. Although the critical point is most important from a mathematical standpoint, its usefulness in gaining physical insight is limited. We have just seen that in an isothermal, thermally driven wind, the critical point marks the point beyond which compressive disturbances cannot be transported upstream (i.e., towards the star). It is no coincidence that the critical point plays this role in the isothermal case. It will, in fact, play such a role whenever information is transported along flow lines by longitudinal waves in which the restoring force has the same acceleration dependence as does the driving force of the wind. There are, however, many circumstances in which such a condition is not met. For example, if information is transported along flow lines by high frequency sound waves that propagate adiabatically in the background isothermal flow, then the sonic point in the flow (the physically relevant point in this case) is located 
where $u^{2}=\gamma v_{t}^{2}$, but the critical point is still located at $u=v_{t}$. Even when the sonic point corresponds to the critical point, information relevant to the acceleration of the wind may be transported upstream beyond this point. For example, if Alfvén waves play a significant role in accelerating the wind, and if the Alfvén speed exceeds the sound speed, then Alfvén waves generated in the region of supersonic flow can transport relevant information upstream into the subsonic region. In a thermally driven wind, electron thermal conduction can modify the wind's driving force by transporting heat upstream in the supersonic flow (in the presence of a positive radial temperature gradient), and in a radiatively driven stellar wind, the transport of electromagnetic radiation (at the speed of light) may have a similar effect.

In general, then, the critical point cannot be expected to mark the point in the flow beyond which information relevant to the acceleration of the wind can be transported upstream. The sonic point, which does not necessarily correspond to the critical point is more likely to fill this role. It is probably safest to view the critical point as a mathematical singularity with limited physical meaning but considerable mathematical significance. Moreover, when we hear a statement like "the wind cannot be thermally driven because the critical point would have to be too near the star" (or "inside the stellar surface", or "too far from the star", etc.), we can be relatively certain that the speaker has avoided an available physical discussion of the problem by appealing to a mathematical platitude.

\section{THE SOLAR WIND: A HOT, TENUOUS WIND FROM A RELATIVELY COOL STAR}

The solar wind is of interest to us, in part, because it is the best observed of all stellar winds. We must keep in mind, though, that its very existence would be difficult to infer from remote-sensing observations alone--i.e., from the sort of observations that are all we have available to us in our studies of other stellar winds. The solar wind is also of interest because it seems to represent a wind driven by two of the mechanisms we consider as candidates for driving massive cool-star winds and because it is frequently cited (appropriately or otherwise) as an analog by theorists attempting to support their ideas for driving mechanisms of a variety of astrophysical flows. In this conference, it has been referred to as an example of a bipolar flow and as an example of a wind that illustrates the combined effects of rotation and magnetic field in driving a flow along the rotation axis. Unfortunately, although we do have much to learn from the solar wind, neither of these particular references is accurate.

We cannot in this space even begin to present a full discussion of the solar wind (cf. the early work of Parker $(1958,1963,1964 \mathrm{a}, \mathrm{b})$, as well as the review by Leer et al. 1982 and references therein), but we can use the solar wind to illustrate some of the general principles outlined in section 2 , and we can also correct the two misconceptions referred to in the preceding paragraph. First, let us consider the concepts introduced in section 2.3. The best observed and most uniform type of solar wind flow is the high speed solar wind stream. These streams are best observed because they are the only type of solar wind for which a coronal source has been unambiguously identified, so that observation of the wind acceleration region is possible. In high speed streams, the asymptotic flow speed of the wind is comparable to the gravitational escape speed at the coronal base: i.e., $u_{\infty}^{2} \approx v_{g o}^{2}$. We can thus infer from the results of section 2.3 that comparable amounts of energy must be supplied to the flow in the subsonic and the supersonic regions. Since the solar atmosphere is strongly gravitationally bound at the coronal base 
(viz., $v_{g o}^{2} \gg 5 v_{t o}^{2} / 2+u_{o}^{2} / 2$ ) the energy flux supplying the required energy to the flow must be transported outward from the coronal base by some mechanism other than advection. Thermal conduction is one such transport mechanism that can be expected to be important, because the corona is so hot $\left(T \gtrless 10^{6} \mathrm{~K}\right)$. Yet, in order for conduction to supply adequate energy to the region of supersonic flow to produce $u_{\infty} \approx v_{g o}$ without the temperature in the subsonic being so high as to produce a larger than observed mass flux, it would be required that radially outward thermal conduction lead to a solar wind temperature that increases radially outward (Leer et al. 1982). Suggestions that this may be the case (e.g., Olbert 1983, Scudder and Olbert 1983) appear at present to have little basis (Lallement et al. 1986, Shoub 1986). It appears, therefore, that some form of mechanical energy transport must supply energy to the region of supersonic flow, and a prime candidate for such a transport mechanism is Alfvén waves (e.g., Hollweg 1978 and references therein). Hence, the solar wind appears likely to be a wave-modified thermally driven wind, with the thermal pressure gradient force lifting the wind out of the solar gravitational field and the waves helping to accelerate the wind to its asymptotic flow speed (through both heating and momentum addition in the supersonic region). This concept of two driving mechanisms working together is one to remember in the following discussions of massive winds from cool stars.

Now, what about the idea that the solar wind is some sort of analog of bipolar flows from other stars? The idea that the solar wind is a bipolar flow apparently comes from the attention received by high-speed solar wind streams and their solar source, coronal holes. During the declining and minimum phases of the solar cycle, well-developed coronal holes usually exist near the solar poles (e.g., articles in Zirker 1977). Hence, during these periods there is a tendency for two (very broad in solar latitude) regions of well-organized high-speed solar wind to dominate the structure of interplanetary space, and the centers of these two wind regions tend to be nearly $180^{\circ}$ apart. It is essential to realize, however, that the solar wind always fills all of interplanetary space (not, for example, just the solar polar regions), and that, if anything, the mass flux density of high speed streams is less than (by as much as $30 \%$ to $50 \%$ ) that of the lower speed, less well organized wind. In any case, the particle density in the high-speed streams is much lower (a factor of two or more) than it is in the lower speed wind. Hence, if we were able to obtain remote, density-sensitive observations of the solar wind (comparable to observations of winds from other stars), we might well infer that most of the solar wind is coming from the equatorial region, but certainly not that it is a bipolar flow from the two polar regions. Unless we substantially modify the usual concept of bipolar flows in astrophysical systems, it seems inappropriate to use the solar wind as an analog for such flows, except insofar as it represents a typical atmospheric expansion from a central gravitating body.

Finally, we may ask whether the solar wind provides supporting evidence for the speculation that bipolar flows can be driven and self-focused by the effects of a magnetic field frozen into a rotating body lying between the oppositely directed flows. The answer is simply that the solar wind has little relevance to this speculation, because beyond a few solar radii the outflowing solar atmosphere begins to take control of the solar magnetic field, stretching it into the classic spiral structure characteristic of a nearly radial fluid flow and a "frozen-in" magnetic field that is tied to the rotating central star (e.g., Parker 1963). The magnetic energy density in the solar wind at the orbit of the earth is only about $1 \%$ of the flow energy density, so the magnetic field has little effect of the flow in the asymptotic region. This particular misuse of the solar wind as an analog for the rotational/magnetic driving of a bipolar flow probably has resulted from the 
inappropriate use of cartoons in place of sound, physically based calculations. Such calculations, if performed, might give us some feeling for whether such a driving mechanism has any hope of explaining some bipolar flows. The absence of such calculations will continue to give free reign to the unbridled imaginations and unsupported speculations of our cartoon-astrophysicists.

\section{MASSIVE, THERMALLY DRIVEN WINDS FROM COOL, LOW-GRAVITY STARS}

In the terminology we are using, a thermally driven wind is one in which the only significant outward force on the expanding atmosphere is exerted by the thermal pressure gradient [viz., $D \approx 0$ in eqn. (2)]. The energy supplied to the atmosphere to maintain the temperature required to produce a sufficiently large pressure gradient force can, in principle, be transported by a variety of mechanisms, including thermal conduction from a hot atmospheric base, a mechanical wave flux, and a radiative energy flux. In practice, however, the massive winds from cool stars seem themselves to be so cool that thermal conduction cannot play a significant role. Furthermore, a radiative equilibrium atmosphere for such cool stars is not hot enough to drive a massive wind through a thermal pressure gradient force alone. Thus, we are left with a mechanical energy flux as the only source (considered up to this time, at least) of energy for heating a thermally driven wind. We must remember, however, that a mechanical energy flux not only heats a wind through its dissipation, but also directly accelerates the wind through the action of a wave pressure gradient force (i.e., $D \neq 0$ ). If this wave force is not negligible in comparison with the thermal pressure gradient force, then we are not dealing with a strictly thermally driven wind, but rather with a wave-modified thermally driven wind, a thermally modified wave-driven wind, or simply a wave-driven wind. This point is touched on again below.

In examining the viability of a thermally driven wind, we can, at first, ignore the mechanism maintaining the atmospheric temperature and simply consider an isothermal (in the subsonic region) wind that exhibits the mass loss rate of an observed wind and a suitable atmospheric base pressure. Then we can calculate the electromagnetic radiation flux (associated with radiative cooling) emanating from such a wind and ask whether such a radiative flux is consistent with observations. Carrying out such an exercise for the range of physical parameters relevant to massive winds from cool, low-gravity stars, it has been found (Holzer and MacGregor 1985) that all such thermally driven winds would have exceedingly large radiative energy flux densities--orders of magnitude larger than the solar radiative energy flux densities at comparable temperatures. Almost all of this radiation arises from a region that extends a few hundredths to a few tenths of a stellar radius above the atmospheric base. The radial extent of this radiating region is orders of magnitude larger than that of the comparable region in the solar atmosphere, and this, of course, accounts for the magnitude of the radiative energy fluxes. Such large radiative energy fluxes would be readily detectable (they are, after all, often comparable in magnitude to the stellar luminosity and occurring at a temperature much higher than the stellar photospheric temperature), but they are not observed.

Watanabe (1981) has tried to get around this difficulty by postulating that the region of elevated atmospheric temperature required for a massive, thermally driven wind is quite narrow, so that the total radiative flux from this region is quite small. Although one can formally obtain thermally driven winds for such an atmospheric temperature structure, Watanabe's proposal has a number of difficulties associated with it (Holzer and MacGregor 1985). One of the most serious is that if such an atmospheric 
temperature structure were produced by the dissipation of any currently known mechanical energy flux, the wave pressure force would dominate the thermal pressure gradient force and the correctly described flow would not be thermally driven.

It seems, therefore, that the thermal pressure gradient force acting alone does not provide a viable mechanism for driving massive winds from cool stars. In addition, the preceding discussion indicates that thermal driving is a very inefficient wind acceleration mechanism when the wind density is sufficiently high that radiative energy losses become substantial. For example, in the study of isothermal winds by Holzer and MacGregor (1985), the energy required to keep the atmosphere sufficiently warm (i.e., to counter radiative losses) was generally several orders of magnitude larger than that required to lift the wind out of the gravitational field of the star and to accelerate it to its asymptotic flow speed.

\section{WAVE-DRIVEN WINDS}

The difficulties encountered in trying to drive massive winds from cool stars with only a thermal pressure gradient force lead us to consider the other type of driving mechanism that may be important in the acceleration of the solar wind: i.e., small to moderate amplitude mechanical waves, specifically hydromagnetic waves (e.g., Hartmann and MacGregor 1980). These waves may be either compressive (e.g., acoustic waves or magnetoacoustic waves: more generally, slow or fast hydromagnetic waves) or noncompressive waves (intermediate hydromagnetic--i.e., Alfvén--waves). Hartmann and MacGregor (1980) have pointed out that the compressive waves would only be able to extend an atmosphere at its base, but not able to lift a massive wind out of the stellar gravitational field, because such waves steepen rapidly into weak shocks and are dissipated within a few pressure scale-heights of the atmospheric base. The non-compressive Alfvén waves, in contrast, are not so readily damped and can do work on an expanding stellar atmosphere over a large enough radial range to drive a quite massive wind.

The momentum addition to a wind associated with a flux of Alfvén waves arises from the wave pressure gradient and is expressed by [cf. eq. (2)]

$$
D=-\frac{1}{\rho} \frac{d}{d r}\left(\frac{<\delta B^{2}>}{8 \pi}\right)=-\frac{1}{\rho} \frac{d}{d r}\left(\frac{1}{2} \rho<\delta v^{2}>\right)
$$

where $\left\langle\delta B^{2}\right\rangle$ and $\left\langle\delta v^{2}\right\rangle$ are the mean-square magnetic field and velocity field of the wave. The net atmospheric heating rate associated with Alfvén wave dissipation and with radiative cooling of the atmosphere can be written in the form [cf. eqn. (3)]

$$
Q=f / \lambda-L_{R}
$$

where the Alfvén wave energy flux density, $f$, is given by

$$
f=\rho<\delta v^{2}>\left(v_{a}+\frac{3}{2} u\right)
$$

$v_{a}=B / \sqrt{4 \pi \rho}$ is the Alfvén speed, and $\lambda$ is the damping length for the waves. The 
damping length in the winds of interest is likely to be determined primarily by ionfrictional damping and the radiative loss rate is probably adequately expressed in terms of an optically thin radiative loss function (Hartman and MacGregor 1980). Because of the strength of radiative losses, the energy balance of a massive wind at chromospheric temperatures $\left(T \approx 10^{4} \mathrm{~K}\right.$ ) can frequently be well approximated by using $L_{R} \approx f / \lambda$ [cf. eqn. (23)] in place of equation (3).

If momentum balance in the flow is dominated by the Alfvén wave pressure gradient force, then (Holzer et al. 1983) the sonic point of the flow occurs near $1.75 R_{*}$, and the mass loss rate is

$$
-\dot{M}_{*} \approx 1.8 \times 10^{-13}\left(\frac{f_{o}}{10^{6} B_{o}}\right)\left(\frac{R_{*}}{R_{\odot}}\right)^{7 / 2}\left(\frac{M_{\odot}}{M_{*}}\right)^{3 / 2} M_{\odot} y^{-1}
$$

where $f_{0}$ (in erg $\mathrm{cm}^{-2} \mathrm{~s}^{-1}$ ) and $B_{0}$ (in gauss) are both evaluated at the atmospheric base $\left(r_{o} \approx R_{*}\right)$. When the Alfvén waves are undamped (i.e., all the wave energy is transferred to the flow through direct acceleration by the wave pressure gradient force) the asymptotic flow speed of the wind is approximately (Holzer et al. 1983)

$$
u_{\infty} \approx v_{g \circ}\left[\frac{8}{7}\left(\frac{v_{a}}{u}\right)_{\circ}+\frac{5}{7}\right]^{1 / 2}
$$

where the subscript $s$ refers to the sonic point. Evidently, an Alfvén wave driven wind will have a mass loss rate comparable to those observed for massive, cool-star winds (cf. section 2.1) even for relatively modest wave energy flux densities at the atmospheric base of $f_{0} \approx 10^{5} B_{0}$ erg $\mathrm{cm}^{-2} \mathrm{~s}^{-1}$. (Note that if the waves are to be small amplitude in the region of subsonic flow, and thus remain undamped by nonlinear processes, then it is required that $B_{o} \gtrless 10$ gauss and that $\left.\left(v_{a} / u\right)_{0} \gg 1\right)$. The asymptotic flow speed, however, is much too large $\left(u_{\infty}^{2} \gg v_{g o}^{2}\right)$ for a wind driven by undamped Alfvén waves. Hartmann and MacGregor (1980) noticed this difficulty and pointed out that damping of Alfvén waves in the region of supersonic flow would significantly reduce the asymptotic flow speed without affecting the mass loss rate. Such damping might be provided by the frictional coupling of atmospheric ions with neutral atoms. Yet, the range of damping rates that produce massive winds with low asymptotic flow speeds is found to be quite small (Holzer et al. 1983), so the wave-driven wind mechanism probably requires some sort of atmospheric self-regulation process to be operative if it is to be the dominant mechanism in driving a substantial number of massive cool star winds. Such a selfregulation mechanism, which would limit the effective wave damping length to an appropriate, narrow range, is still being sought.

\section{RADIATIVELY DRIVEN WINDS}

Our understanding of radiatively driven winds comes primarily from extensive studies of massive, very high speed $\left(u_{\infty}^{2} \gg v_{g o}^{2}\right)$ winds from early-type stars (e.g., review by Cassinelli and MacGregor 1986, and references therein). The radiative flux in these hot stars exhibits a significant energy flux in the ultraviolet portion of the electromagnetic spectrum, and since the atmosphere has many strong resonance lines in this same part of the spectrum, the radiation field is well coupled to the expanding atmosphere and can add a 
substantial amount of momentum to a stellar wind. The situation is quite different for cool stars, in which the radiative energy flux peaks in the red or near-infrared, and the strong atmospheric resonance lines occur in the visible and ultraviolet. The resulting poor spectral match implies that the potential effectiveness of radiative driving of massive winds from these cool stars can be realized only if some other opacity source can provide for efficient coupling of the radiation field to the wind. Two possible sources of opacity are provided by molecules and dust in the stellar atmosphere, but at present only the effects of dust have been considered extensively (e.g., review by Goldberg 1984, and references therein). We can illustrate the sort of role dust (or molecules) might play in driving a massive, cool-star wind with a very simple analysis, from which we can also infer some of the problems that need to be addressed before we understand the actual roles dust and molecules play.

The force per unit mass associated with radiative driving of a stellar wind can be written in the form [cf. eqns. (2) and (8)]

$$
D=\frac{v_{o o}^{2} r_{o}}{2 r^{2}} \Gamma(r)
$$

Evidently, $\Gamma$ is just the ratio of the magnitudes of the local gravitational force and the local radiative force. In general, $\Gamma$ must be considered to be a function of the various macroscopic (and perhaps microscopic) parameters of the expanding stellar atmosphere, but for the purpose of the present simplified discussion, we take $\Gamma$ to be a function of radial distance. This approach allows us to draw some general conclusions about radiative driving of winds, but it would not be appropriate, for example, to apply such an approach to the study of phenomena like the radiative amplification of hydromagnetic waves.

In the models of dust-driven winds that might be appropriate to those cool stars with dusty atmospheres, the atmospheric dust and gas are generally so strongly frictionally coupled that radiative momentum transfer to the dust can be treated as momentum addition to the atmosphere as a whole (i.e., to both dust and gas, the latter of which comprises most of the stellar wind mass). For these models, therefore, the radial variation of $\Gamma$ is determined by effects such as grain condensation and destruction, spectral redistribution of the emergent stellar radiation field through interaction with the dust, and radial variation of the dust density through atmospheric expansion. Let us consider a particularly simple case, in which there is no dust near the star, and dust is formed in a narrow radial range centered at radial distance $r=r_{c}$. The reason for labelling this distance $r_{c}$ is that it also marks the critical point of the flow in our simple representation, as can be seen by considering a modified form of the isothermal wind equation (18), which includes the effects of radiative momentum addition:

$$
\left(u^{2}-v_{t}^{2}\right) \frac{1}{u} \frac{d u}{d r}=\frac{2}{r}\left[v_{t}^{2}-\frac{1}{4} v_{g}^{2}(1-\Gamma)\right]
$$

In the case at hand, we assume $4 v_{t}{ }^{2}<v_{g}{ }^{2}$ and $\Gamma<<1$ in $r<r_{c}$, and $\Gamma>1$ in $r>r_{c}$. Evidently, then, the zero of the right side of equation (18) occurs at $r=r_{c}$ and this is where the flow must be sonic. An examination of equation (20) indicates that moving the critical point inward from its position $r_{c t}$ for a thermally driven wind to the position $r_{c d}$ 
where dust is formed leads to an increase in the mass loss rate by a factor of

$$
\frac{\dot{M}_{* d}}{\dot{M}_{* t}}=\left(\frac{r_{c d}}{r_{c t}}\right)^{2} \exp \left[2\left(\frac{r_{c t}}{r_{c d}}-1\right)\right] \text {. }
$$

The magnitude of the radiatively driven mass loss can be expressed simply in terms of critical point parameters:

$$
-\dot{M}_{* d}=4 \pi \rho_{c} \quad v_{t c} r_{c}^{2}
$$

The exponential increase of the mass loss rate $\left(-\dot{M}_{* d}\right)$ with decreasing $r_{c d}$ [cf. eqn. (29)] reflects the exponential falloff of density in the subsonic region of the wind and the consequent exponential increase of critical point density $\left(\rho_{c}\right)$ as the critical point is moved inward [cf. eqn. (30)].

If we try to argue that the principal driving mechanism of winds from cool, lowgravity stars with dusty atmospheres is radiation pressure on dust grains, then we encounter the difficulty that this mechanism tends to produce relatively small mass loss rates, as long as the dust formation region is at relatively large radial distances (i.e., $\left.r_{c d} \approx 10 R_{*}\right)$. This difficulty could be overcome if a region of sudden grain formation were to occur very near the star (viz., $r_{c d} \lessgtr 2 R_{*}$ ), but the bulk of observational evidence seems to weigh against such a hypothesis, at least at present (e.g., Goldberg 1984). Alternatively, if grains were formed over a broad radial region near the star, or if "clean" grains near the star were to become "dirtier" with increasing radial distance (Draine 1981), resulting in a gradual increase of grain opacity over a broad radial region, then the atmospheric scale height in the subsonic region $\left(r<r_{c}\right)$ could be significantly increased, and a substantial mass loss rate could be radiatively driven even if the critical point were to occur relatively far from the $\operatorname{star}\left(r_{c} \lesssim 10 R_{*}\right)$. Unfortunately, we will not be in a position to evaluate such ideas until our understanding of grain formation. in stellar atmospheres is substantially improved.

Another difficulty that is encountered in trying to attribute the driving of winds from cool, low-gravity stars to radiation pressure alone is that the atmospheric opacity structure must have a very special configuration to avoid producing large asymptotic flow speeds. To illustrate this point, consider again the simple case in which grain formation occurs in a narrow radial region. The asymptotic flow speed can be calculated from equation (12), if we first note that [cf. eqn. (27)]

$$
\begin{gathered}
\int_{r_{0}}^{r_{c}} d r D=O, \\
\int_{r_{c}}^{\infty} d r D \approx \frac{v_{g o}^{2} r_{o}}{2 r_{c}}\left(\frac{\Gamma_{c}}{1+\beta_{c}}\right),
\end{gathered}
$$

where $\beta_{c}=\langle-d(\ln \Gamma) / d(\ln r)\rangle$ is an average over the region just beyond $r_{c}$. Combining equations (12) and (31), 


$$
u_{\infty}^{2} \approx v_{g o}^{2}\left(\frac{r_{o}}{r_{c}}\right)\left(\frac{\Gamma_{c}}{1+\beta_{c}}-1\right)
$$

If we wish to have a substantial mass flux (i.e., $r_{c} / r_{o}$ is not too large), to ensure that the mass flux is lifted out of the gravitational field (i.e., $u_{\infty}^{2}>0$ ), and to have a small asymptotic flow speed (i.e., $u_{\infty}^{2}<<v_{g o}^{2}$ ), then we see that $r_{c}, \beta_{c}$, and $\Gamma_{c}$ must be very finely tuned. Is there some sort of atmospheric self-regulation mechanism operating that can produce such a fine tuning? This, of course, is just the question that we had to ask in the consideration of wave-driven winds and that we must ask again in the context of shock-driven winds.

\section{SHOCK-DRIVEN WINDS}

Many of the stars which are surrounded by enough dust to provide strong dynamical coupling between the radiation field and the atmosphere also exhibit regular pulsations that can drive large-amplitude shock waves outward through the atmosphere. Because such shock waves deposit both heat and momentum in the stellar atmosphere, they may play an important role in driving stellar winds from pulsating stars, especially the Miras (e.g., Willson and Hill 1979; Wood 1979; references in Holzer and MacGregor 1985). Indeed, because of the frequent coexistence of dust and pulsations, it is possible that in some stars radiation-driving and shock-driving combin'e to produce the observed stellar winds (e.g., Wood 1979; Jura 1984). Let us first, however, consider the shock-driving mechanism in isolation.

Although a shock-driven wind is inherently non-steady, we can gain a feeling for the basic physical effects important in such a wind by considering a time-averaged view, which involves casting an approximate theoretical description in the steady flow framework that we have used up to now. Following our usual procedure, we ask first how we might formulate momentum and heat addition terms arising from the outward propagation of quasi-periodic shocks. When a shock front passes through a fluid element in a stellar atmosphere, that fluid element experiences rapid heating and acceleration. In a relatively strong shock, the heating is almost entirely dissipative (i.e., non-adiabatic or irreversible), which is to say that compressive (adiabatic) heating is small in comparison with the total heating associated with shock front passage. Hence, most of the heat produced will not be lost in the expansive cooling of the fluid element throughout the broad rarefaction region behind the shock front. The average heating rate owing to shock passage is thus

$$
Q_{8} \approx \rho(\delta u)^{2} / \tau
$$

where $\delta u$ is the shock speed in the local average rest frame of the atmosphere (moving at speed $\langle u\rangle$ ), and $\tau$ is the pulsation period. We can similarly approximate the momentum addition rate by

$$
D_{\bullet} \approx \delta u / \tau
$$

Of course, in the dense stellar atmospheres we are considering, radiative cooling behind 
the shock front can play an important role in counteracting shock heating. In the limit of very strong radiative cooling, the shocks can be described as isothermal (the above description was for adiabatic shocks) and the atmosphere is then taken to be in radiative equilibrium. In this case, although the shock heating effects become negligible, the momentum addition [eqn. (34)] still plays a role in accelerating the wind. This contrasts sharply with the adiabatic case, in which (as long as $\delta u /\langle u\rangle$ is large) heating is the major source of energy addition to the wind.

Theoretical descriptions have been developed for the two extreme cases of adiabatic and isothermal shocks, and the results of the two descriptions are drastically different. Using an adabatic shock description (in which the wind is largely thermally driven) Wood (1979) calculated a mass loss rate some four orders of magnitude larger than the observationally inferred rate for the Mira variable being modelled, and some ten orders of magnitude larger than the mass loss rate calculated using an isothermal shock description. One finds in an a posteriori evaluation of these calculations that that radiative cooling rate far exceeds the shock heating rate in the wind driven by adiabatic shocks, while just the opposite is true for the isothermal case. Thus, neither description comes close to providing an energetically consistent description of the wind. Evidently, there is much work to do before a clear statement can be made about the importance of shockdriving in the winds of pulsating stars, and at least some of this work (involving a more realistic treatment of energy balance) needs to be carried out before one can make a suitable evaluation of the idea that shock-driving and radiation-driving may work in concert to produce the massive winds from pulsating stars with dusty atmospheres.

\section{SUMMARY}

The goal of this paper has been to provide a framework for thinking about the various physical processes that may play significant roles in driving the winds of cool, lowgravity stars. We have concentrated on four mass loss mechanisms and described them in the context of steady, radial, spherically symmetric flow, but the basic physical character of these mechanisms remains essentially the same when they are invoked in the context of asymmetric, nonsteady mass loss, and when they act in concert with each other. Hence, an understanding of the relatively simple models described here should provide a sound basis for understanding the relatively complex phenomena that are actually observed.

The general discussion of mass, momentum, and energy balance (cf. section 2), on which the descriptions of the simple models were based, provides us with the ability to articulate one of the most difficult problems encountered in trying to understand the driving of massive winds from cool, low-gravity stars. Observations seem to indicate that the asymptotic flow speeds of these winds are generally much less than the gravitational escape speed at the base of the atmosphere (i.e., $u_{\infty}^{2}<<v_{g o}^{2}$ ). If this inference is correct, then we can conclude from the arguments given in section 2 that most of the energy required to drive the wind goes into lifting the wind out of the stellar gravitational field, and that most of this energy is supplied to the region of subsonic flow. The small fraction of the energy supplied by the region of supersonic flow is tightly constrained by the requirement that the asymptotic flow speed be small, but positive. This tight constraint, which seems to apply to a broad range of stellar winds, implies the existence of some sort of atmospheric self-regulation (associated with the wind driving mechanism(s)) that finely tunes the amount of energy deposited in the region of supersonic flow. One of the difficult problems we face is, thus, to explain how a driving 
mechanism or combination of driving mechanisms can lead to this sort of atmospheric self-regulation in a broad range of massive winds from cool, low-gravity stars.

ACKNOWLEDGMENTS. I am grateful to Keith MacGregor for useful discussions and for comments on the manuscript. I would also like to thank Lorraine Hori for preparing the camera-ready manuscript.

\section{REFERENCES}

Cassinelli, J.P. and MacGregor, K.B. 1986, in Physics of the Sun, ed. P.A. Sturrock, T.E. Holzer, D. Mihalas, and R.K. Ulrich (Chicago: University of Chicago Press), p. 47.

Draine, B.J. 1981, in Physical Processes in Red Giants, ed. I. Iben and A. Renzini (Dordrecht: Reidel), p. 317.

Goldberg, L. 1984, in CNRS-NASA Monograph Series on Nonthermal Phenomena in the Stellar Atmosphere (Washington, D.C.: NASA), Ch. 7.

Hartmann, L. and MacGregor, K.B. 1980, Ap. J., 242, 260.

Hollweg, J.V. 1978, Rev. Geophys., 16, 689.

Holzer, T.E., Flå, T., and Leer, E. 1983, Ap. J., 275, 808.

Jura, M. 1984, Ap. J., 282, 200.

Lallement, R., Holzer, T.E., and Munro, R.H. 1986, J. Geophys. Res., 91, 6751.

Leer, E. and Holzer, T.E. 1980, J. Geophys. Res., 85, 4681.

Leer, E., Holzer, T.E., and Flå, T. 1982, Space Sci. Rev., 33, 161.

Olbert, S. 1983, in Solar Wind 5, NASA Conf. Publ. 2280, 149.

Parker, E.N. 1958, Ap. J., 128, 664.

- 1963, Interplanetary Dynamical Processes (New York: Interscience).

1964a, Ap. J., 139, 72.

1964b, Ap. J., 139, 93.

Scudder, J.D. and Olbert, S. 1983, in Solar Wind 5, NASA Conf. Publ 2280, 163.

Shoub, E. 1986, in preparation.

Watanabe, T. 1981, Publ. Astron. Soc. Japan, 33, 679.

Willson, L.A. and Hill, S.J. 1979, Ap. J., 228, 854.

Wood, P.R. 1979, Ap. J., 227, 220.

Zirker, J. (Ed.) 1977, Coronal Holes and High Speed Solar Wind Streams, Colorado Associated University Press, Boulder. 